\title{
The Measurement of Charge for Induction-Based Fluidic MALDI Dispense Event and Nanoliter Volume Verification in Real Time
}

\author{
Brent Hilker, ${ }^{a}$ Kevin J. Clifford, ${ }^{\text {a }}$ Andrew D. Sauter Jr, ${ }^{\text {b }}$ \\ Andrew D. Sauter 3rd, ${ }^{b}$ and Julie P. Harmon ${ }^{a}$ \\ a Department of Chemistry, University of South Florida, Tampa, Florida, USA \\ ${ }^{\mathrm{b}}$ Nanoliter LLC, Henderson, Nevada, USA
}

This study preliminarily investigates whether nanoliter volumes of concentrated polar liquids and organic monomers launched to targets using induction based fluidics (IBF) can be verified through the real time charge measurements. We show that using a nanoliter IBF dispensing device and nanocoulomb meter, charge measurements made on nanoliter drops in real time are correlated with surface area following Gauss's Law. We infer the "induction only" formation of the double layer showing the ability to determine nanoliter volumes, nearly instantaneously, in real time. We discuss the implications that these observations may have for on improving/monitoring MALDI quantitation and its quality control. (J Am Soc Mass Spectrom 2009, 20, 1064-1067) (c) 2009 American Society for Mass Spectrometry

$\mathrm{H}$ erein we used an induction based fluidics (IBF) nanoliter, microliter syringe [1]. In IBF, a charge is induced on the liquid by passing the fluid through an electric field [2, 3], inductively, not conductively as in electrospray ionization (ESI). In IBF there are no Faradaic processes, only capacitance based ones, unlike ESI. The physics behind IBF reveals [2, 3] that, unlike piezoelectric, sound, or any other technologies that are applied to transport liquids at low volumes, IBF kinetically launches drops to targets, as it dynamically directs the liquids to targets, and-as we show here-measures them on arrival, in real time.

One major IBF application, nanoliter (nL) depositions for the production of matrix-assisted laser desorption/ ionization mass spectrometry (MALDI-MS) plates, has been shown to increase 10-fold in signal-to-noise ratios for both bradykinin measurements and analysis precision [4]. Nanoliter-IBF depositions also produce a major increase in MALDI sensitivity and reproducibility for synthetic polymers [5] (PMMA, PEG, and PS), even with polymers with MN values greater than 90,000 units. Yergey has also observed up to a 100-fold increase in analysis sensitivity [6] for a major class of proteins, tubulins. These and similar observations on the analytes in the Applied Biosystems (ABI) 4700 Standard [4] show significant improvement (increases

Address reprint requests to Prof. Julie P. Harmon, University of South Florida, Department of Chemistry, 4202 E. Fowler Ave., Tampa, FL 33620. E-mail: harmon@cas.usf.edu of around 5-, 10-, to 100-fold) in sensitivity and a 3 to $20 \%$ increase in reproducibility using nanoliter IBF depositions for proteins, peptides, and synthetic polymers. These enhancements in a wide range of molecules and mass ranges in both positive-ion reflectron mode and negative-ion linear mode indicate that nL-IBF deposition improves sensitivity across many MALDI applications. The ability to verify a dispense event and its magnitude in real time may aid quantitative quality control (QC) on MALDI measurements, irrespective of whether they are produced on a one-at-a-time basis or via high-throughput applications on robotic systems.

IBF has many MS and non-MS applications because the simple technique can be appended to common laboratory devices that are used for routine nanoliter sample handling from syringes, pipettes, chips, pumps, and other fluid movement devices including tissue and liquid chromatography (LC)/MALDI devices. The ability to accurately measure and to verify the volume deposited per event by IBF in real time could further aid the ability to QC and to improve MALDI quantitation. The ability to easily manipulate small-volume solvents or solutes to targets also has applications in the areas of green chemistry and in biological MALDI with limited sample sizes [4]. Applications of nL-IBF exist in nontouch dispensing (micro-, nano-, and picoliter samples), in parallel LC/MALDI, in defense homeland security [7], chemistry, for desorption electrospray ionization/ direct analysis in real time (DESI/DART) standardization and applications [8], DNA/RNA sample preparation [9], 
and in thin-layer chromatography (TLC) applications [10]. With IBF nanoliter quantities of viscous liquid, potential applications for human serum or whole human blood can be flown to MALDI targets [11].

To explain IBF, in a flowing or stop-flow laminar system, the volume dispensed is described by the Hagen-Poiseuille equation [12], as follows:

$$
V=\left(\left(\pi r^{4} P\right) / 8 \eta 1\right) t
$$

This law states that the volume of fluid $(V)$ that flows down a small-diameter capillary tube per unit of time $(t)$ is proportional to the fourth power of the radius of the tube $(r)$, the pressure pushing the fluid down the tube $(P)$, and inversely proportional to the length of the tube $(l)$ and the viscosity of the fluid $(\eta)$. Droplets produced can be placed in an electric field and become inductively energized. In these nearly perfect spheres the charge exists primarily on the surfaces $[13,14]$. The nanoliter device creates an exact volume mechanically in one mode. Purely electrokinetic IBF operation is also possible, but is not addressed here.

When a droplet is placed in an electric field $(E)$ the droplet becomes charged $(q)$ and it experiences a force (F) [15], expressed as

$$
F=q E
$$

This force launches the drop to a target with excellent accuracy and precision in a manner analogous to that used by Millikan [15] in his work determining the charge of the electron. This force is the same force used in mass spectrometers [16] to accelerate and focus gas-phase ions in a vacuum. Like gas-phase ions, charged drops can be accelerated and directed in a dynamic manner to targets producing useful results. Further details are beyond the scope of this presentation. Refer to Sauter [3,17] for a more complete discussion.

Gauss's Law applied to the electrostatic charge on a sphere can be expressed as [18]

$$
q=k S A
$$

The charge on a drop $(q)$ is proportional to the surface area (SA) with constant $k$. Constant $k$ is the product of the electric field $(E)$ and the electric permittivity of the substance $(\varepsilon)$. The volume $(V)$ relationships for a sphere of radius $r$ are shown below. The measurements of the charge on drops produced by the IBF dispensing device at high potential were performed.

$$
\begin{aligned}
& \mathrm{V}=4 / 3 \pi \mathrm{r}^{3} \\
& \mathrm{SA}=4 \pi \mathrm{r}^{2} \\
& \mathrm{SA}=4 \pi\left(3 / 4 \mathrm{~V} \pi \mathrm{r}^{2}\right)^{2 / 3} \\
& \mathrm{q}=\mathrm{k}\left[4 \pi\left(3 / 4 \mathrm{~V} \pi \mathrm{r}^{2}\right)^{2 / 3}\right]
\end{aligned}
$$

\section{Experimental}

\section{Materials}

$\mathrm{NaCl}$ and $12 \mathrm{M} \mathrm{HCl}$ (Fischer Chemicals, Fair Lawn, NJ, USA). Purified 2-hydroxyethyl methacrylate (2-HEMA) was donated by Benz Research and Development (Sarasota, FL, USA).

\section{Apparatus and Procedure}

The Nanoliter LLC IBF device (217 Garfield Drive, Henderson, NV 89074, USA) was connected to a programmable controller that determined volume through displacement. No residual liquid was noted remaining on the fused silica (FS) needle tip nor was any excess liquid seen being drawn into the FS needle from the Hamilton syringe chamber. A $10.0 \mu \mathrm{L}$ removable needle (RN) syringe was inserted using polyamine-coated FS capillary needles $(20 \mathrm{~cm}$ long, i.d. $50 \mu \mathrm{m}$, o.d. $150 \mu \mathrm{m})$. A Keithley 6514 programmable electrometer (Keithley Instruments, Cleveland, $\mathrm{OH}$, USA) was set to measure in nanocoulombs $(\mathrm{nC})$ using a copper triaxial cable. A copper Faraday cup $(2 \times 1 \times 1 \mathrm{~cm}$, designed by the University of South Florida) measured electric potential between the droplet and earth ground. A Faraday cage was constructed with aluminum screen (1.5- $\mathrm{cm}$ mesh).

The electrometer was set to the $\mathrm{nC}$ mode. The copper was contact connected and completed electrical connections between the Faraday cup, triaxial cable, and the electrometer, thus eliminating any charge capacitance buildup from the near contact of two dissimilar metals [19]. The Faraday cup was suspended in midair, not in contact with the Faraday cage. Since the magnitude of induced charge on droplets dispensed into the Faraday cup is very small and the energy is high, environmental electric fields and electromagnetic interference (EMI) affect the accuracy of the measured charge [20] requiring the use of a Faraday cage. A schematic of this apparatus is presented in the supplemental section, which can be found in the electronic version of this article. This setup provided nearly instantaneous real time charge measurements.

Electrolyte solutions ( $\mathrm{NaCl}$ and $\mathrm{HCl}), 0.33 \mathrm{M}, 0.166$ $\mathrm{M}, 0.091 \mathrm{M}$ in deionized $\mathrm{H}_{2} \mathrm{O}$ and 2-HEMA (pure monomer) were launched into the Faraday cup. Four trials were conducted for each size. The rate of ambient charge buildup was measured so that the ambient charge was no greater than $0.001 \mathrm{nC} / \mathrm{s}$. The mean and standard deviation were calculated for each volume and concentration. The cup was grounded between each shot.

\section{Results and Discussion}

After Gauss and Thomson and Iribarne's [21] model the surface area is used as the dependent variable in plots. Surface areas (SAs) in plots of solutions ranged from 24 to $263 \mathrm{~nm}^{2}(11-400 \mathrm{~nL})$. Figure 1 shows charge mea- 


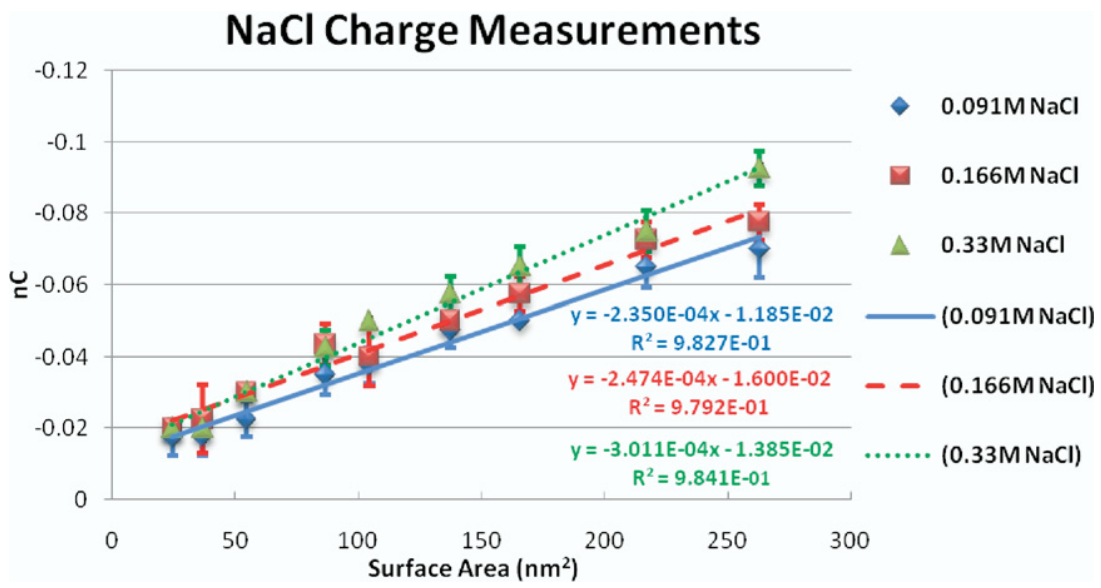

Figure 1. Charge $(\mathrm{nC})$ versus surface area $\left(\mathrm{nm}^{2}\right)$ shows linear relation for all concentrations of $\mathrm{NaCl}$ : $\Delta \mathrm{NaCl} 0.33 \mathrm{M} ; \mathrm{NaCl} 0.166 \mathrm{M} ; \mathrm{NaCl} 0.091 \mathrm{M}$. Error bars represent standard deviation for charge.

surements for $\mathrm{NaCl}$ at varied concentrations. The experiments reveal a strongly correlated, potentially linear relationship between SA and charge on droplets, in agreement with previous studies $[2,14]$ and with Gauss's Law. The charge can also be correlated to volume, nonlinearly, as shown in Figure 2, showing $\mathrm{NaCl}$ at varied concentrations. $\mathrm{HCl}$ showed the same linear relationship of charge to $\mathrm{SA}$ as $\mathrm{NaCl}$. Figures for $\mathrm{HCl}$ relating SA to charge and volume to charge are available in the supplemental section.

Next we examined the surface charge upon a pure organic monomer (2-HEMA) used in polymerization reactions. Results obtained for surface charge on droplets $53-305 \mathrm{~nm}^{2}(37-500 \mathrm{~nL})$ were similar to the electrolyte solutions ( $\mathrm{HCl}$ and $\mathrm{NaCl})$. There is a high correlation of charge to surface areas for 2-HEMA. Real time volume verification through charge measurement is possible for both electrolyte $(\mathrm{NaCl}$ and $\mathrm{HCl})$ solutions and pure organic monomer (2-HEMA). Figures for
2-HEMA showing SA to charge and volume to charge are available in the supplemental section.

All $\mathrm{nC}$ versus surface area graphs show nonzero intercepts, which we interpret as being attributed to a charging mechanism other than being related to the drop surface volume. A number of potential sources can contribute to this-from air particulates to the inductive charging of the apparatus itself, which may be the case here because the intercepts are numerically similar. The difficulties in measuring low currents at high energy are well known [22]. These nonzero intercepts are not surprising, but of course they limit the ultimate sensitivity of the technique to measure/verify volume deposition. We note that this prototype device was not optimized to limit EMI effects between the device and the sample. As such, the nonzero intercepts are not surprising.

Different slopes were anticipated and dilutions were run to see how charge varied with different volumes for different liquids and thus different surface areas. That

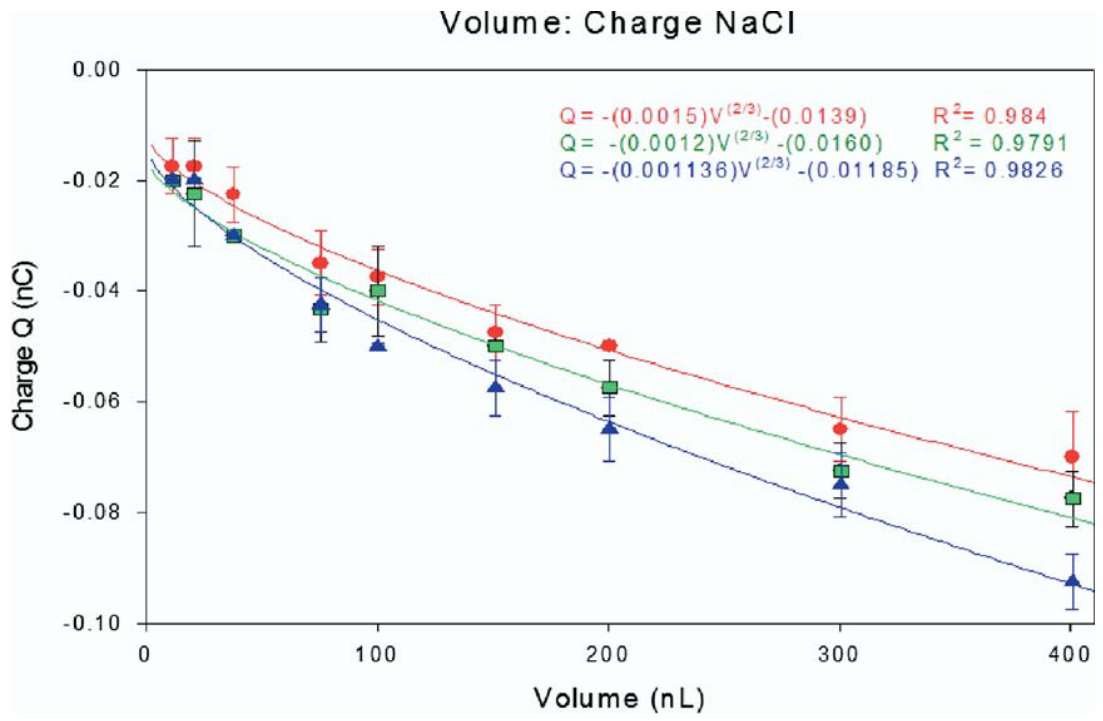

Figure 2. Charge $(\mathrm{nC})$ versus volume $\left(\mathrm{nm}^{3}\right) \mathrm{NaCl}: \mathbf{\Delta} \mathrm{NaCl} 0.33 \mathrm{M} ; \mathbf{\square a C l} 0.166 \mathrm{M} ; \bullet \mathrm{NaCl} 0.091 \mathrm{M}$. Error bars represent standard deviation for charge. 
surface area strongly correlates with volume, as shown, indicates that: (1) the IBF force arises from the formation of the double layer in the electric field and (2) it is capacitance based, i.e., it was without Faradaic processes that can change the liquid. The fact that charge varied from a few different liquids and dilutions shows that the approach is generally applicable, although some nonideal behavior may have been from highly concentrated solutions.

In Figure 1 we had hoped to observe slopes related to sample concentrations. Rather, the charges, especially at the lowest volumes, were similar, irrespective of the liquid. In these cases we could measure differences within, but not between, samples. We attribute that observation to the fact that these solutions are of high concentration where nonideal liquid solution behavior is common. This nonideal behavior can affect the electrical double layer (EDL) - an electrostatic attraction of ions and counter ions in the liquid to preferentially populate the region near the surface [23]. It is also known that the thickness of the double layer, whose formation is inferred in IBF, can shrink dramatically with increasing concentration, a phenomenon known as double-layer compression [24]. It is this property of the double layer that explains the fact that the slopes do not differ significantly, although at the highest volumes the anticipated order is observed for the mean values [24].

Different slopes were observed from different electrolyte solutions of the same concentration. This may be attributed to the physical packing of the ions in the electret double layer. The difference in occupied volumes between $\mathrm{Na}$ and $\mathrm{H}$ cations may have led to a different packing at the double layer and thus different electret double-layer charge.

\section{Conclusion}

Our experiments have shown that a nL-IBF device can launch a drop to a target. When the drop arrives to the target its charge can be measured in real time. Because charge is highly correlated with surface area according to Gauss's Law, we infer the formation of the double layer and thus the ability to determine the deposition of nanoliter solution volumes in real time.

The importance of requesting a known low volume of liquid (via the digital syringe controller) and verifying that the specified amount has reached the target (via the measurement of charge) with a simple device has enumerable applications from drug delivery to quantitative MALDI because the exact volume of the liquid transferred to targets, which is not always guaranteed in low-volume transfers, can now be verified for quality control and other purposes on slow or fast timescales in real time. With feedback, verified real time control could be achieved to enhance the accuracy of liquid dispensing and/or liquid treatment applications (e.g., LC/MALDI).

Finally, because IBF can be applied to dispense viscous liquids, from monomers as shown here and elsewhere [1, 3-6], to samples including human serum, heparinzed whole human blood, or other body fluids, our approach could afford a valuable QC mechanism for MALDI assays of these important liquids. Verifying the dispensing event and its volume in real time could allow molar correction or alignment via charge measurements and might realize the goal of a routinely quantitative MALDI.

\section{Appendix A Supplementary Material}

Supplementary material associated with this article may be found in the online version at doi:10.1016/ j.jasms.2009.01.015.

\section{References}

1. Sauter, A. D. Precise Electrokinetic Delivery of Minute Volumes of Liquid. U.S. Patent 6149815,2000 ; and U.S. pending patents of Sauter, A. D.: 60/574,104; 60/759,787; 60/881,532; and 61/011,178.

2. Delgado, A. V.; Gonzalez-Caballero, F.; Hunter, R. J.; Koopal, L. K.; Lyklema, J. Measurement and Interpretation of Electrokinetic Phenomena. J. Colloid Interface Sci. 2007, 309, 194-224.

3. Sauter, A. D., Jr. The Nanoliter Syringes (ASMS MALDI poster). Am. Laboratory 2007, February.

4. Tu, T.; Sauter, A. D., 3rd; Sauter, A. D., Jr.; Gross, M. L. Improving the Signal Intensity and Sensitivity of MALDI Mass Spectrometry by Using Nanoliter Spots Deposited by Induction-based Fluidics. J. Am. Soc. Mass Spectrosc. 2008, 19, 1086-1090.

5. Hilker, B.; Clifford, K. J.; Sauter, A. D., Jr.; Sauter, A. D., 3rd; Gauthier, T.; Harmon, J. P. Electric Field Enhanced Sample Preparation for Synthetic Polymer MALDI-TOF Mass Spectrometry via Induction Based Fluidics (IBF). Polymer 2009, 50, 1015-1024.

6. Yergey, A. L. National Institutes of Health, Bethesda, MD. Personal communication, 2008.

7. Colquhoun, D. R.; Schwab, K. J.; Cole, R. N.; Halden, R. U. Detection of Norovirus Capsid Protein in Authentic Standards and in Stool Extracts by Matrix-Assisted Laser Desorption Ionization and Nanospray Mass Spectrometry. Appl. Environ. Microbiol. 2006, 72, 2749-2755.

8. Fernandez, F. M.; Cody, R. B.; Green, M. D.; Hampton, C. Y.; McGready, R.; Sengaloundeth, S.; White, N. J.; Newton, P. N. Characterization of Solid Counterfeit Drug Samples by Desorption Electrospray Ionization and Direct-Analysis-in-Real-Time Coupled to Time-of-Flight Mass Spectrometry. ChemMedChem 2006, 1, 702-705.

9. Ding, C. Qualitative and Quantitative DNA and RNA Analysis by Matrix-Assisted Laser Desorption/Ionization Time-of-Flight Mass Spectrometry. Methods Mol. Biol. 2006, 336, 59-71.

10. Rozylo, J. K.; Berezkin, V. G.; Malinowska, I.; Jamrozek-Manko, A Technical Problems with the Application of Solutes to Chromatographic Plates in TLC. J. Planar Chromatogr. 2001, 14, 272-276.

11. Datta, S., Conlisk, A. T. Role of Multivalent Ions and Electrical Double Layer Overlap in Electroosmotic Nanoflows. In Proceedings of the 47th AIAA Aerospace Sciences Meeting, January 2009, Orlando, FL.

12. Hitzer, E. Early Works on the Hagen-Poiseuille Flow. Memoirs of the Faculty of Engineering, Univ. of Fukui 2001, 49, 45-53.

13. Kebarle, P.; Tang, L. From Ions in Solutions to Ions in the Gas Phase. The Mechanism of Electrospray Mass Spectrometry. Anal. Chem. 1993, 65, 972-986.

14. Song, S. P.; Li, B. Q. A Hybrid Boundary/Finite Element Method for Simulating Viscous Flows and Shapes of Droplets in Electric Fields. Int. J. Comput. Fluid Dynamics 2001, 15, 293-308.

15. Millikan, R. A. On the Elementary Electric Charge and the Avogadro Constant. Phys. Rev. II 1913, 2, 109

16. Amster, I. J. Fourier Transform Mass Spectrometry. J. Mass Spectrom. 1996, 31, 1325-1337.

17. Sauter, A. D., Jr.; Sauter, A. D., 3rd. Electric Zip Tips ${ }^{\mathrm{TM}}$ Preliminary Results. J. Assoc. Lab. Automation 2002, 7, 52-55.

18. Halliday, D.; Resnick, R. Physics. Wiley: New York, 1962

19. Ferrante, J.; Smith, J. R. Theory of the Bimetallic Surface. Phys. Rev. B. 1985, 31, 3427-3434.

20. Amin, M. S. Advanced Faraday Cage Measurements of Charge, ShortCircuit Current and Open-Circuit Voltage. M.S. Thesis. Massachusetts Institute of Technology: Cambridge, MA, September 2004.

21. Thomson, B. A.; Iribarne, J. V. Field Induced Ion Evaporation from Liquid Surfaces at Atmospheric Pressure. J. Chem. Phys. 1979, 71, 4451-4463.

22. Low Level Measurements Handbook: Precision DC Current, Voltage, and Resistance Measurements, 6th edition. Keithley Instruments, Inc.: Cleveland, $\mathrm{OH}, 2008 ;$ pp $2.44-2.66$.

23. Seraglia, R.; Teatino, A.; Traldi, P. MALDI Mass Spectrometry in the Solution of Some Forensic Problems. Forensic Sci. Int. 2004, 146S, S83-S85.

24. Han, K. N. Fundamentals of Aqueous Metallurgy. Society for Mining, Metallurgy and Exploration: Littleton, CO, 2002; pp 25-27. 